\title{
Performance Analysis of Adaptive Hybrid Nonlinear Preprocessors for Impulsive Noise Mitigation over Power-Line Channels
}

\author{
Khaled M. Rabie and Emad Alsusa \\ Microwave and Communication Systems (MCS) Group, \\ School of Electrical and Electronic Engineering, \\ The University of Manchester, Manchester, UK, M13 9PL, \\ Email: \{khaled.rabie, e.alsusa $\} @$ manchester.ac.uk
}

\begin{abstract}
Impulsive noise (IN) over power-lines can significantly corrupt communication signals. To diminish its effect, a nonlinear preprocessor is usually applied at the receiver's frond-end to blank or clip the incoming signal when it exceeds a certain threshold. Applying a combination of blanking and clipping in a hybrid fashion is characterized by two thresholds $T_{1}$ and $T_{2}\left(T_{2}=\alpha T_{1}\right)$, where $\alpha$ is a scaling factor. Previous studies assumed a fixed value for the scaling factor and found that optimizing the threshold $T_{1}$ is the key to improve performance. In contrast to the existing work, in this paper we show that the performance of the hybrid technique is sensitive not only to the threshold but also to the scaling factor, and in light of this we propose to enhance the capability of this technique by optimizing the two parameters. System Performance is evaluated mathematically in terms of the probability of missed blanking/clipping $\left(P_{m}\right)$, probability of IN identification $\left(P_{i}\right)$ and the symbol error rate (SER) performance. In all our investigations, simulation results are provided to validate the analysis. Results reveal that the proposed scheme is superior in terms of minimizing $P_{m}$ and maximizing $P_{i}$ which consequently results in improving SER performance.
\end{abstract}

Index Terms-Blanking, clipping, hybrid, impulsive noise, powerline communications (PLC), probability of miss, probability of identification, SER performance.

\section{INTRODUCTION}

Over the recent decades, the rising dependence on communications has increased remarkably and owing to the advances in communication, modulation techniques as well as signal processing, it has become feasible to exploit power-lines for highspeed communication with data rates comparable to that provided by wired networks and wireless LANs [1]-[3]. The fact that power-line networks were not designed to carry communication signals, such signals can suffer from severe degradation. Thus, in order to improve the reliability of power-line communication (PLC) systems, it is of paramount importance to overcome several inherent challenges such as the varying impedance of the wiring, high levels of frequency-dependent attenuation and the noise [4]. Contrary to many other communication channels, noise over power-lines cannot be described as additive white Gaussian noise (AWGN), it is rather categorized into impulsive noise (IN) and background noise [5]-[8]. The former, however, has at least $10-15 \mathrm{~dB}$ power spectral density higher than that of the latter, [8], making IN the most crucial element influencing communication signals over PLC channels.

Orthogonal frequency division multiplexing (OFDM) systems have been widely proposed for PLC [9] since not only they are very efficient in combating the channel frequency-selectivity but can also provide a code diversity effect which is able, somewhat, to reduce the impact of IN [10]. Interestingly enough, however, this advantageous effect could turn into a disadvantage if IN energy exceeds a certain threshold. In such scenarios, additional IN mitigating methods must be employed such as the application of nonlinear devices at the receiver's front-end to clip or/and zero the received signal with it exceeds a predetermined threshold value(s). In general, there are three common nonlinear preprocessors reported in the literature namely: blanking, clipping and hybrid (joint blanking and clipping), which are widely used in practice because of their simplicity and ease of implementation [11]-[13]. Determining the appropriate blanking and/or clipping threshold(s), in fact, remains the key for achieving best performance. For instance, overlooking the IN signals will worsen the probability of missed blanking/clipping $\left(P_{m}\right)$ and hence performance will deteriorate whereas perfect recognition of noise pulses will leverage the probability of IN identification $\left(P_{i}\right)$ resulting in more reliable communications. In order to enhance these probabilities, the optimal threshold must be used. In general, the hybrid technique was shown to offer the best performance compared to the other nonlinear techniques [13]. In this scheme, two thresholds are set $T_{1}$ and $T_{2}$ to clip or blank the incoming signal when it exceeds these thresholds, respectively, and are related by the scaling factor $(\alpha)$ as $T_{2}=\alpha T_{1}$.

Most work on this topic assumes a fixed scaling factor; this method will be classified here as the conventional hybrid technique. In contrast, in this paper, we show that the performance of the hybrid technique is not purely threshold-dependent but is also sensitive to the scaling factor. Therefore, the contribution of this paper is as follows. First, the minimum $P_{m}$ and maximum $P_{i}$ of the optimized system are analyzed. Then the impact of various nonlinear preprocessors on the signal constellation at the output of the OFDM demodulator is investigated for two modulation schemes. Finally, the symbol error rate (SER) performances is evaluated. To provide more quantitative characterization of the proposed system, in all investigations, we include results for blanking, clipping, conventional hybrid techniques as well as that for a typical OFDM receiver with no nonlinear preprocessing at the receiver's front-end. Throughout the paper, simulation results are provided to validate our analysis. The results reveal that the adaptive approach is able to considerably minimize $P_{m}$ and maximize $P_{i}$. It is also found that the proposed system always establishes the lower bound performance in terms of 


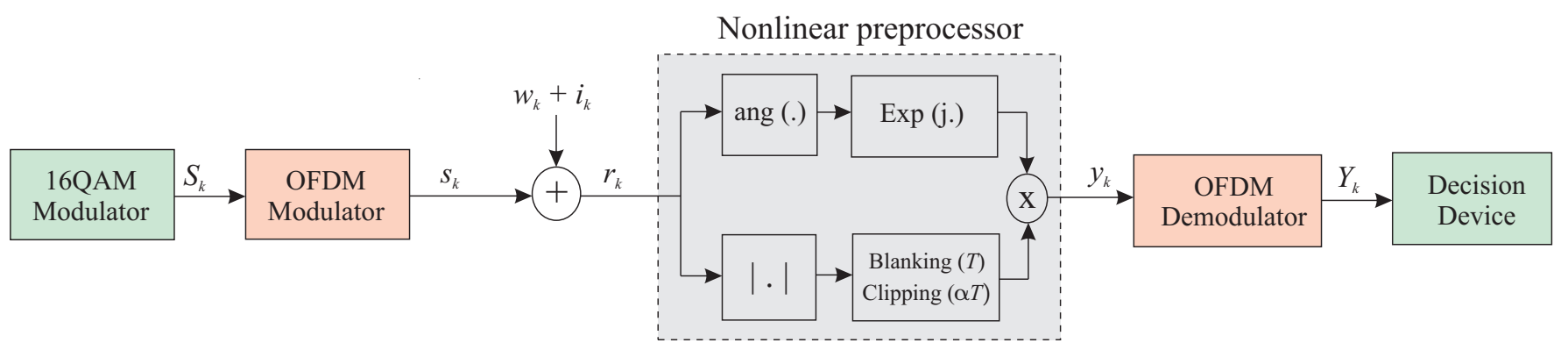

Figure 1: System diagram with nonlinear preprocessors at the receiver.

SER performance and that higher modulation schemes are more sensitive to IN.

The rest of the paper is organized as follows. In Section II, the system model is introduced. Section III investigates the output SNR, the threshold and scaling factor optimization problem as well as $P_{m}$ and $P_{i}$ performance for the optimized systems. Signal constellations for different modulation schemes are investigated in Section IV whereas Section V is dedicated to study the SER performance. Finally, conclusions are drawn in Section VI.

\section{SySTEM MODEL}

The system model under consideration is shown in Fig. 1. First the information bits are mapped into 16-quadrature amplitude modulation (16-QAM) base-band symbols $S_{k}$. Then, these symbols are passed through an OFDM modulator to produce a time domain signal

$$
s(t)=\frac{1}{\sqrt{N}} \sum_{k=0}^{N-1} S_{k} e^{\frac{j 2 \pi k t}{T_{s}}}, \quad 0<t<T_{s}
$$

where $N$ is the number of sub-carriers and $T_{s}$ is the active symbol interval. In this work and for simplicity we adopt the two component mixture-Gaussian noise model in which IN is modeled as a Bernoulli-Gaussian random process [10], [14] and is given by

$$
n_{k}=w_{k}+i_{k} \quad k=0,1,2, \ldots, N-1
$$

where

$$
i_{k}=b_{k} g_{k}, \quad k=0,1,2, \ldots, N-1
$$

$n_{k}$ is the total noise component, $w_{k}$ is the AWGN, $i_{k}$ is the IN, $g_{k}$ is complex white Gaussian noise with mean zero and $b_{k}$ is the Bernoulli process with probability $\operatorname{Pr}\left(b_{k}=1\right)=p$, where $p$ denotes the IN probability of occurrence. The probability density function (PDF) of the total noise can be expressed as

$$
P_{n_{k}}\left(n_{k}\right)=p_{0} \mathcal{G}\left(n_{k}, 0, \sigma_{0}^{2}\right)+p_{1} \mathcal{G}\left(n_{k}, 0, \sigma_{1}^{2}\right)
$$

while $\mathcal{G}($.$) is the Gaussian PDF, p_{0}=(1-p), p_{1}=p, \sigma_{0}^{2}=$ $\sigma_{w}^{2}$ and $\sigma_{1}^{2}=\sigma_{w}^{2}+\sigma_{i}^{2}$. The variances $\sigma_{w}^{2}$ and $\sigma_{i}^{2}$ denote the AWGN and IN powers which define the input SNR and signalto-impulsive noise ratio (SINR) as $\mathrm{SNR}=10 \log _{10}\left(1 / \sigma_{w}^{2}\right)$ and $\mathrm{SINR}=10 \log _{10}\left(1 / \sigma_{i}^{2}\right)$, respectively.
Under perfect synchronization condition, the received signal is expressed as

$$
r_{k}=\left\{\begin{array}{ll}
s_{k}+w_{k}, & \mathcal{H}_{0} \\
s_{k}+w_{k}+i_{k}, & \mathcal{H}_{1}
\end{array} \quad k=0,1, \ldots, N-1\right.
$$

where $s_{k}=s\left(k T_{s} / N\right) ; s_{k}, w_{k}$ and $i_{k}$ are assumed to be mutually independent. The null hypothesis $\mathcal{H}_{0}$ implies the absence of IN, $P\left(\mathcal{H}_{0}\right)=(1-p)$, whereas the alternative hypothesis $\mathcal{H}_{1}$ implies the presence of IN, $P\left(\mathcal{H}_{1}\right)=p$. In order to reduce the effect of IN, one of the following nonlinear preprocessors is applied at the front-end of the receiver

- Clipping

$$
y_{k}=\left\{\begin{array}{ll}
r_{k}, & \left|r_{k}\right| \leq T_{1} \\
T_{1} e^{j \arg \left(r_{k}\right)}, & \left|r_{k}\right|>T_{1}
\end{array} \quad k=0,1, \ldots, N-1\right.
$$

where $T_{1}$ is the clipping threshold.

- Blanking

$$
y_{k}=\left\{\begin{array}{ll}
r_{k}, & \left|r_{k}\right| \leq T_{2} \\
0, & \left|r_{k}\right|>T_{2}
\end{array} \quad k=0,1, \ldots, N-1\right.
$$

where $T_{2}$ is the blanking threshold.

- Conventional Hybrid

$$
y_{k}= \begin{cases}r_{k}, & \left|r_{k}\right| \leq T_{1} \\ T_{1} e^{j \arg \left(r_{k}\right)}, & T_{1}<\left|r_{k}\right| \leq T_{2} \quad k=0,1, \ldots, N-1 \\ 0, & \left|r_{k}\right|>T_{2}\end{cases}
$$

where $T_{2}=1.4 T_{1}$.

- Adaptive Hybrid

$$
y_{k}= \begin{cases}r_{k}, & \left|r_{k}\right| \leq T \\ T e^{j \arg \left(r_{k}\right)}, & T<\left|r_{k}\right| \leq \alpha T \quad k=0,1, \ldots, N-1 \\ 0, & \left|r_{k}\right|>\alpha T\end{cases}
$$

where $r_{k}$ and $y_{k}$ are the input and the output of the nonlinear devices, respectively, and $\alpha>1$. Clearly, these devices only process the amplitude of the received signal leaving its phase unmodified. In order to maximize system performance, the threshold(s), or the threshold and the scaling factor in case of adaptive hybrid, should be carefully selected.

\section{Performance Optimization}

To begin with, the output SNR of the four nonlinear preprocessors (6), (7), (8) and (9), can generally be expressed as [13]

$$
\mathrm{SNR}_{\text {out }}=\frac{2 K_{o}^{2}}{E_{\text {out }}-2 K_{o}^{2}}
$$




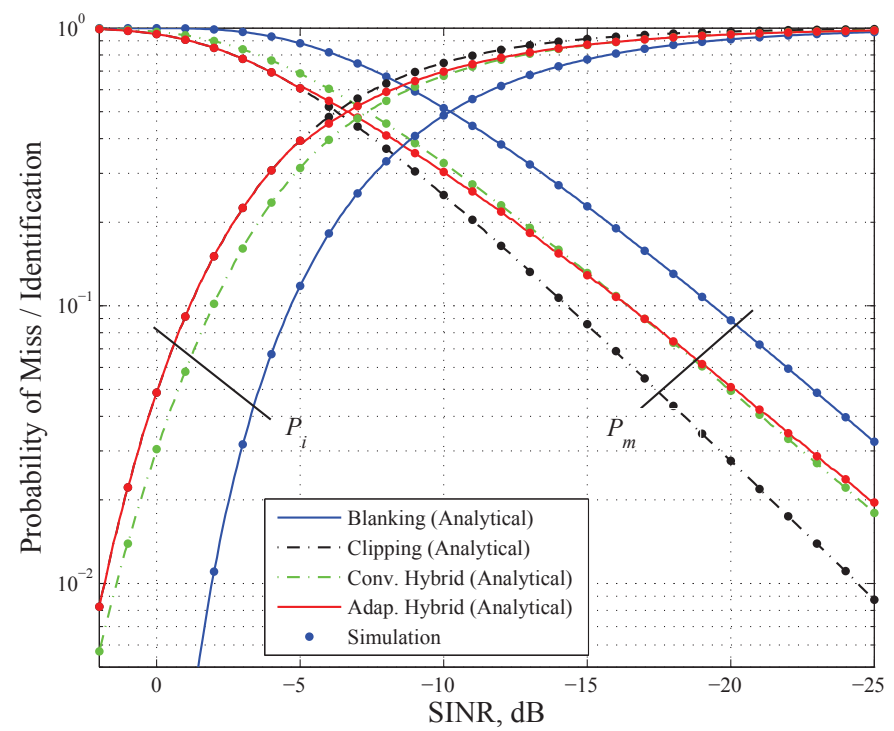

(a) $p=0.01$

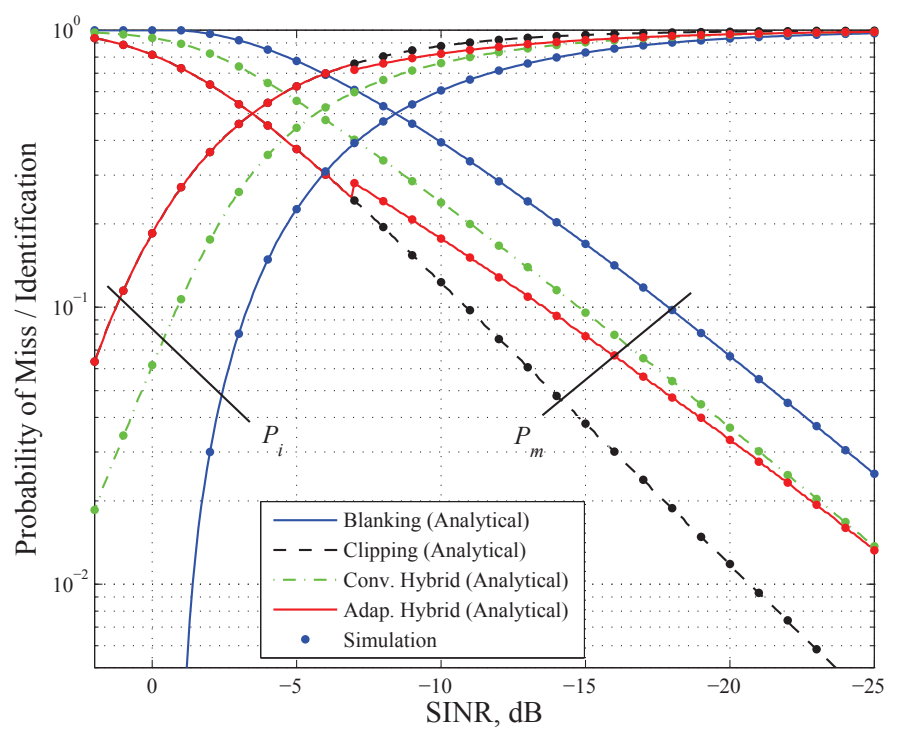

(b) $p=0.1$

Figure 2: Probability of miss and probability of identification versus SINR for the blanking, clipping, conventional hybrid and adaptive hybrid systems when input $\mathrm{SNR}=25 \mathrm{~dB}$ for $p=0.01$ and 0.1 .

where $K_{O}$ is a real constant and $E_{\text {out }}$ is the total signal power at the output of the nonlinear device. These parameters are derived in [13] for the blanking, clipping and conventional hybrid techniques whereas for the adaptive hybrid system, $K_{o}$ and $E_{\text {out }}$ are presented in [15]. It should be mentioned here that for the typical OFDM system, i.e. no blanking or/and clipping takes place, the output SNR can simply be given as

$$
\operatorname{SNR}_{\text {out }}(T \rightarrow \infty, \alpha \rightarrow \infty,)=10 \log _{10}\left(\frac{\sigma_{s}^{2}}{\sigma_{w}^{2}+p \sigma_{i}^{2}}\right)
$$

In order to optimize the output SNR of the OFDM nonlinear preprocessing based systems, it is more convenient to rewrite (10) as

$$
\left(\mathrm{SNR}_{\text {out }}\right)^{-1}=\frac{E_{\text {out }}}{2 K_{\text {o }}^{2}}-1
$$

It is evident that the optimal threshold and optimal scaling factor cannot be expressed in closed-forms hence only numerical results will be obtained by satisfying the following argument

$$
\min _{T, \alpha}\left\{\frac{E_{\text {out }}}{K_{o}^{2}}\right\}
$$

The optimized threshold and scaling factor values are then used to calculate $P_{m}$ and $P_{i}$. These probabilities are highly dependent on the IN characteristics and are defined as follows. $P_{m}$ is the probability that the affected signals are not blanked and is expressed as $P_{m}=P\left(A_{r}<T \mid \mathcal{H}_{1}\right) P\left(\mathcal{H}_{1}\right)$; whereas $P_{i}$ is the probability of correctly blanking the contaminated samples and is calculated as $P_{i}=P\left(A_{r}>T \mid \mathcal{H}_{1}\right) P\left(\mathcal{H}_{1}\right)$. Since the amplitude of the received signal has Rayleigh distribution; $P_{m}$ and $P_{i}$, for the optimized system, are calculated by [16]

$$
P_{m}=p\left(1-\exp \left(-\frac{T_{o p t}^{2}}{2\left(\sigma_{s}^{2}+\sigma_{w}^{2}+\sigma_{i}^{2}\right)}\right)\right)
$$

$$
P_{i}=p \exp \left(-\frac{T_{o p t}^{2}}{2\left(\sigma_{s}^{2}+\sigma_{w}^{2}+\sigma_{i}^{2}\right)}\right),
$$

respectively, where $T_{o p t}$ is the optimal threshold corresponding to the optimized scaling factor which is obtained numerically by satisfying (13). Fig. 2 shows some numerical results of (14) and (15) as a function of SINR along with simulation results for $p=0.01$ and 0.1. For comparison's sake, $P_{m}$ and $P_{i}$ for the optimized blanking, clipping and conventional hybrid based systems are also included. From this point onward, unless it is stated otherwise, our simulations are based on an OFDM system with $N=256$ sub-carriers, 16-QAM modulation, $\sigma_{s}^{2}=$ $(1 / 2) \mathbb{E}\left[\left|s_{k}\right|^{2}\right]=1, \sigma_{w}^{2}=(1 / 2) \mathbb{E}\left[\left|w_{k}\right|^{2}\right], \sigma_{i}^{2}=(1 / 2) \mathbb{E}\left[\left|i_{k}\right|^{2}\right]$, input $\mathrm{SNR}=25 \mathrm{~dB}$.

The first observation one can see from Fig. 2 is that $P_{m}$ and $P_{i}$ are inversely proportional. It can also be noted that as the noise becomes more impulsive, these probabilities improve and the opposite is true when noise pulses become smaller. This is intuitively justified by the fact that when SINR approaches zero the amplitude of the OFDM and IN signals become more comparable leading to inaccurate recognition of the IN whereas when the noise is very impulsive it becomes easily distinguished and, hence can be perfectly detected. In addition, it is observed that the adaptive hybrid scheme has better $P_{m}$ and $P_{i}$ performance than that of the conventional hybrid system when (SINR $>-15 \mathrm{~dB}$ ) and this enhancement increases as the pulse probability becomes higher. Furthermore, it is evident that when SINR is very low (SINR $<-15 \mathrm{~dB}$ ) both systems perform similarly irrespective of the IN probability.

It is to be emphasized at this point that $P_{m}$ and $P_{i}$ cannot be stand-alone measures of the system performance but a good tradeoff of both is more crucial. This encourages us to have a closer look at the signal constellation after the OFDM demodulator and investigate the SER performance more insight. 


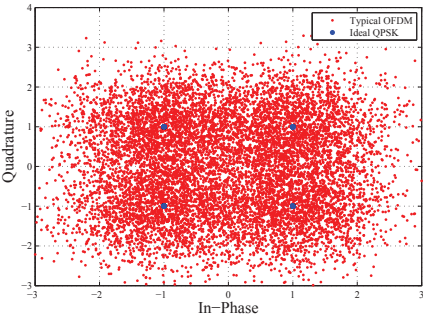

(a) Typical OFDM receiver

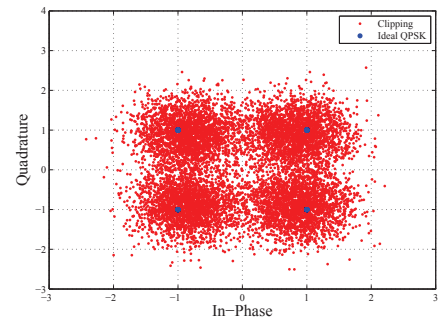

(c) Clipping

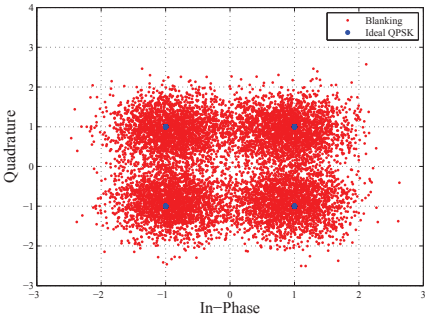

(b) Blanking

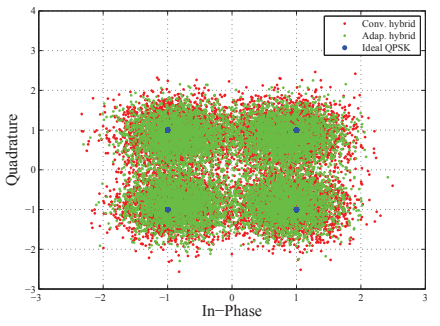

(d) Conventional / adaptive hybrid

Figure 3: Signal constellation points after the OFDM demodulator for QPSK modulation in the presence of IN for the typical OFDM receiver, blanking, clipping and hybrid system when SINR $=-7 \mathrm{~dB}, p=0.1$ and input $\mathrm{SNR}=$ $25 \mathrm{~dB}$.

\section{CONSTEllations' SENSITIVITY Discussion}

In this section we investigate the impact of blanking, clipping, conventional hybrid and adaptive hybrid techniques on the signal constellation at the output of the OFDM demodulator. Two different modulation schemes are considered here namely, QPSK and 16-QAM. However, to achieve communication systems with very high levels of spectral efficiency, very dense QAM constellations is usually employed. For example, the current HomePlug AV2 power-line standard supports 4096-QAM modulation (12 bits/symbol) [17] which is 4 times higher than the previously released HomePlug AV standard [18], [19]. Using higher-order QAM without increasing the BER requires increasing the signal energy, reducing noise, or both. In our investigation in this section we keep the signal power unchanged and examine the impact of reducing IN using the four nonlinear preprocessors on the QPSK and 16-QAM constellation points.

Fig. 3 illustrates the constellation diagram of the QPSK signal in the presence of IN after the demodulator for the typical OFDM receiver, blanking, clipping, conventional hybrid and adaptive hybrid systems. The noise characteristics adopted in this section are input $\mathrm{SNR}=25 \mathrm{~dB}, \mathrm{SINR}=-7 \mathrm{~dB}$ and $p=0.1$. A noiseless ideal QPSK signal will have a constellation consisting of distinct points $\{ \pm 1\}$ symmetric around the real and imaginary axes - the blue points in Fig. 3. However, as can be seen from Fig. 3(a) IN intensively dislocates the constellation points. It is observed that when a nonlinear device is applied, Figs. 3(b)-(d), the scattering is constrained considerably and four clusters centered around the original four points are formed. It can also be noted that the adaptive hybrid technique provides slightly better constellation precision than the conventional hybrid system. It is worthwhile mentioning the fact that the lower the scattering the more accurate the detection decision becomes.

It is commonly known that by moving to a higher-order constellation, it becomes possible to transmit more bits per symbol. However, if the mean energy of the constellation is kept fixed for

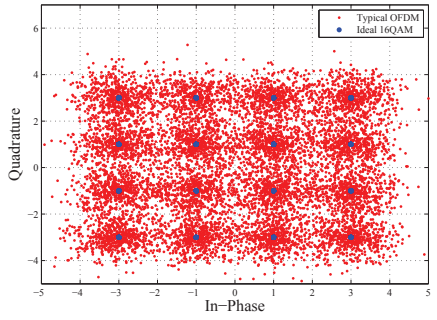

(a) Typical OFDM receiver

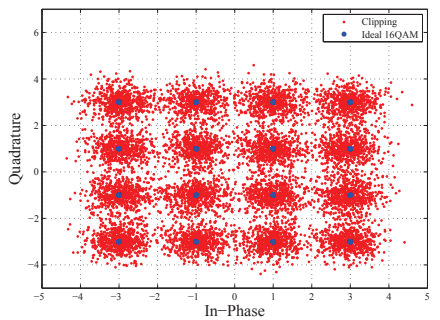

(c) Clipping

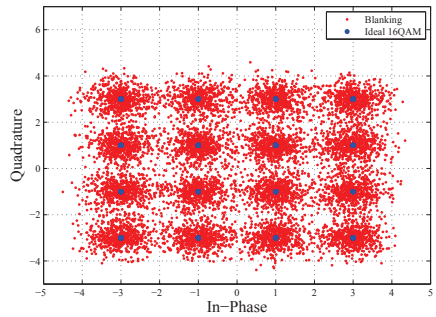

(b) Blanking

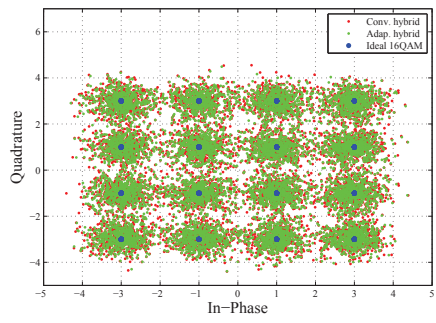

(d) Conventional / adaptive hybrid
Figure 4: Signal constellation points after the OFDM demodulator for 16QAM modulation in the presence of IN for the typical OFDM receiver, blanking, clipping and hybrid when SINR $=-7 \mathrm{~dB}, p=0.01$ and input SNR $=25 \mathrm{~dB}$.

a fair comparison, which is the case here, the points will be closer together and will therefore be more susceptible to noise. This can be observed by comparing Fig. 3(a) and Fig. 4(a) even though the IN probability is 10 times lower compared to that of the QPSK system. In addition, it is evident that the improvement offered by the nonlinear devices becomes insignificant as the constellation size increases. Furthermore, it is interesting to see from Fig. 3(d) and Fig. 4(d) that the adaptive hybrid system provides negligible enhancement relative to that obtained with the QPSK system. For more quantitative and meaningful results, we now assess the symbol error probability of the two aforementioned QAM constellations in different IN environments.

\section{Symbol ERror Rate Performance}

This section is dedicated to analyze the SER performance of the proposed system. To analytically evaluate the bit/symbol error rate performance in OFDM receivers with nonlinear preprocessors, two assumptions are usually made. 1) The noise at the output of the OFDM demodulator approaches Gaussian distribution if the number of sub-carriers is sufficiently large ( $N=8192$ subcarriers in this evalution) [20]-[22]. 2) Sufficient number of samples within an OFDM symbol should be contaminated with IN such that $N p \gg 1$. With this in mind, the conventional SER prediction techniques can be used. Figs. 5 and 6 depict the SER performance versus SINR for the blanking, clipping, conventional hybrid and adaptive hybrid systems when $p=0.1$ and 0.03 for QPSK and 16-QAM modulation, respectively. Again to provide a comparative analysis, we have included the SER results of the typical OFDM receiver on both plots. The analytical results are straightforwardly obtained by substituting the maximum achievable output SNR calculated from (10) and (11) into [23]

$$
S_{e}=1-\left[1-2\left(1-\frac{1}{\sqrt{L}}\right) Q\left(\sqrt{\frac{3 \mathrm{SNR}_{\text {out }}}{L-1}}\right)\right]^{2}
$$




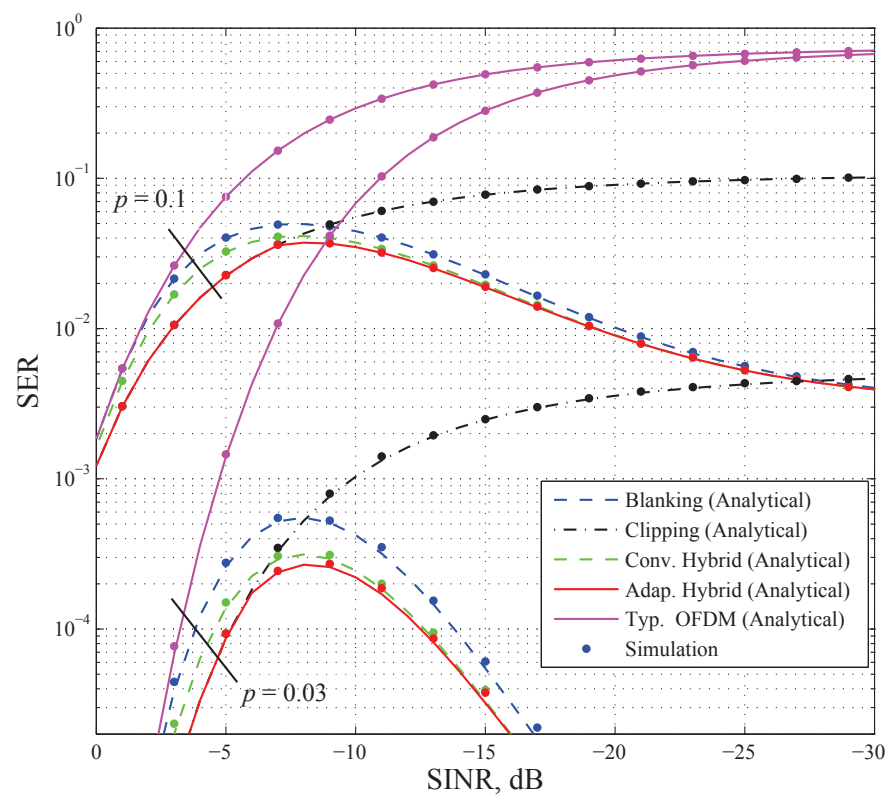

Figure 5: SER performance versus SINR for the blanking, clipping, conventional hybrid, adaptive hybrid and the typical OFDM receiver with various IN probabilities, input SNR $=25 \mathrm{~dB}$ and QPSK modulation.

where $L$ is the constellation order which is 4 or 16 in this case of QPSK and 16-QAM, respectively, and $Q($.$) is the Gaussian$ $Q$-function defined as

$$
Q(x)=\frac{1}{\sqrt{2 \pi}} \int_{x}^{\infty} \exp \left(-\frac{x^{2}}{2}\right) \mathrm{dx}
$$

From these figures it is shown that the analytical results correlate well with the simulated ones for all the systems under consideration. One can see that the adaptive hybrid technique establishes the lower bound performance in all IN scenarios whereas the typical OFDM receiver has the worst performance in comparison to the other systems. It is also clearly visible that when the IN probability is high, the SER improvement obtained with the adaptive hybrid scheme, compared to the conventional hybrid system, is larger and becomes insignificant when $p$ is low in which case the latter technique could be applied instead since it is simpler. In general, and as anticipated, the systems with 16-QAM modulation are more sensitive to IN compared to the QPSK system for the reasons previously discussed. In both constellations, however, it is worth stressing that the proposed system enhances the performance at the expense of slightly higher computational complexity at the receiver since two parameters are optimized. Besides, it is interesting to note that the SER performance of the typical OFDM receiver follows that of the systems with nonlinear preprocessors when SINR $\gtrsim-4 \mathrm{~dB}$ and it diverges as SINR becomes smaller. This is simply because the OFDM demodulator provides a code diversity effect which is able, to some extent, to mitigate some of the IN impact; i.e. IN energy is spread out over $N$ sub-carriers causing less over all interference. On the other hand, when SINR $\rightarrow-\infty$ the SER approaches 1 regardless of the IN probability of occurrence. This agrees with the results concluded in [10] that as IN becomes more impulsive, the spreading of the IN energy over $N$ sub-carriers is no longer advantageous and it rather becomes deleterious since all the $N$ symbols are affected with high levels of interference.

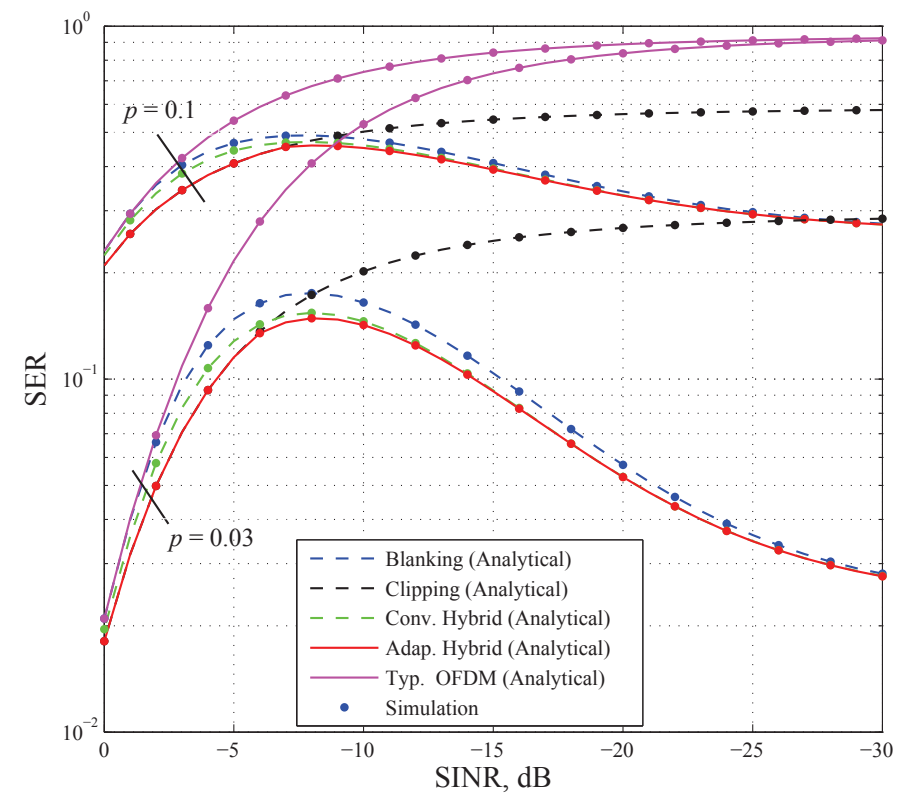

Figure 6: SER performance versus SINR for the blanking, clipping, conventional hybrid, adaptive hybrid and the typical OFDM receiver with various IN probabilities, input SNR $=25 \mathrm{~dB}$ and 16-QAM modulation.

This implies that in such environments the exploitation of a nonlinear preprocessor becomes even more appealing.

\section{Vi. CONCLUSION}

In this paper we have proposed to enhance the capability of the hybrid technique by jointly optimizing the threshold and the scaling factor. The probability of miss, probability of identification and SER performances were evaluated for the optimized blanking, clipping conventional hybrid and adaptive hybrid based systems in various noise environments. All the results in this paper were validated through computer simulations. Results show that the adaptive hybrid technique is able to considerably minimize the probability of miss, maximize the probability of identification as well as improving the SER performance. It is also found that the proposed scheme establishes the lower bound performance of the OFDM nonlinear preprocessing based systems.

\section{REFERENCES}

[1] H. Latchman and L. Yonge, "Power line local area networking," IEEE Commun. Mag., vol. 41, no. 4, pp. 32-33, Apr. 2003.

[2] N. Pavlidou, A. J. H. Vinck, J. Yazdani, and B. Honary, "Power line communications: state of the art and future trends," IEEE Commun. Mag., vol. 41, no. 4, pp. 34-40, Apr. 2003.

[3] S. Galli, A. Scaglione, and K. Dostert, "Broadband is power: internet access through the power line network," IEEE Commun. Mag., vol. 41, no. 5, pp. 82-83, May 2003.

[4] D. Anastasiadou and T. Antonakopoulos, "Multipath characterization of indoor power-line networks," IEEE Trans. Power Del., vol. 20, no. 1, pp. 90-99, Jan. 2005.

[5] D. Middleton, "Canonical and quasi-canonical probability models of class A interference," IEEE Trans. Electromagn. Compat., vol. EMC-25, no. 2, pp. 76-106, May 1983.

[6] ㄴ. "Non-gaussian noise models in signal processing for telecommunications: new methods an results for class A and class B noise models," IEEE Trans. Inf. Theory, vol. 45, no. 4, pp. 1129 -1149, May 1999.

[7] M. G. Sanchez, L. de Haro, M. C. Ramon, A. Mansilla, C. M. Ortega, and D. Oliver, "Impulsive noise measurements and characterization in a UHF digital TV channel," IEEE Trans. Electromagn. Compat., vol. 41, no. 2, pp. 124 -136, May 1999. 
[8] M. Zimmermann and K. Dostert, "Analysis and modeling of impulsive noise in broad-band powerline communications," IEEE Trans. Electromagn. Compat., vol. 44, no. 1, pp. 249-258, Feb. 2002.

[9] E. Del Re, R. Fantacci, S. Morosi, and R. Seravalle, "Comparison of CDMA and OFDM techniques for downstream power-line communications on low voltage grid," IEEE Trans. Power Del., vol. 18, no. 4, pp. 1104-1109, Oct. 2003.

[10] M. Ghosh, "Analysis of the effect of impulse noise on multicarrier and single carrier QAM systems," IEEE Trans. Commun., vol. 44, no. 2, pp. 145-147, Feb. 1996.

[11] K. S. Vastola, "Threshold detection in narrow-band non-gaussian noise," IEEE Trans. Commun., vol. COM-32, no. 2, pp. 134-139, Feb. 1984.

[12] A. D. Spaulding, "Locally optimum and suboptimum detector performance in a non-gaussian interference environment," IEEE Trans. Commun., vol. 33, no. 6, pp. 509-517, Jun. 1985.

[13] S. V. Zhidkov, "Analysis and comparison of several simple impulsive noise mitigation schemes for OFDM receivers," IEEE Trans. Commun., vol. 56, no. 1, pp. 5-9, Jan. 2008

[14] B. Choi and L. Hanzo, "Adaptive WHT aided QAM for fading channels subjected to impulsive noise," IEEE Commun. Lett., vol. 17, no. 7, pp. 1317-1320, Jul. 2013.

[15] K. M. Rabie and E. Alsusa, "Threshold and scaling factor optimization for enhancing impulsive noise cancellation in PLC systems," in Proc. IEEE Global Commun. Conf. (GLOBECOM), Dec. 2014, pp. 2977-2982.

[16] E. Alsusa and K. Rabie, "Preprocessing-based impulsive noise reduction for power-line communications," IEEE Trans. Power Del., vol. 29, no. 4 , pp. 1648-1658, Aug. 2014.

[17] HomePlug AV2 Technology. HomePlug Powerline Alliance, 2012.

[18] HomePlug AV White Paper. HomePlug Powerline Alliance, 2005.

[19] M. Hazen, "The technology behind HomePlug AV powerline communications," Computer, vol. 41, no. 6, pp. 90-92, Jun. 2008.

[20] J. Haring and A. J. H. Vinck, "Iterative decoding of codes over complex numbers for impulsive noise channels," IEEE Trans. Inf. Theory, vol. 49, no. 5, pp. 1251-1260, May 2003.

[21] S. Wei, D. L. Goeckel, and P. E. Kelly, "A modern extreme value theory approach to calculating the distribution of the peak-to-average power ratio in OFDM systems," in Proc. IEEE Int. Conf. Commun. (ICC), vol. 3, Apr. 2002, pp. 1686-1690.

[22] P. Banelli and S. Cacopardi, "Theoretical analysis and performance of OFDM signals in nonlinear AWGN channels," IEEE Trans. Commun., vol. 48, no. 3, pp. 430-441, Mar. 2000.

[23] J. Proakis, Digital Communications. 4th ed. New York: McGraw-Hill, 2001. 